\title{
VARIABILIDADE ESPACIAL E TEMPORAL DO FÓSFORO, POTÁSSIO E DA PRODUTIVIDADE DE UMA LAVOURA CAFEEIRA
}

\author{
GABRIEL A. E S. FERRAZ ${ }^{1}$, FÁBIO M. DA SILVA², LUIS C. C. CARVALHO ${ }^{3}$, \\ MARCELO DE C. ALVES ${ }^{4}$, BRUNO C. FRANCO ${ }^{5}$
}

\begin{abstract}
RESUMO: A agricultura de precisão surge como uma importante ferramenta para melhorar o gerenciamento da produção cafeeira, mostrando que o conhecimento de determinadas características do solo, associado à resposta de produtividade do cafeeiro, pode facilitar a aplicação localizada e racional dos insumos, com resultados ambientais e econômicos positivos. Desta forma, o presente trabalho objetivou utilizar ferramentas de agricultura de precisão aliadas a ferramentas da geoestatística para avaliar as variáveis fósforo, potássio e produtividade do cafeeiro, em três safras agrícolas, por meio de análises dos semivariogramas e de mapas de isolinhas interpolados por krigagem, e demonstrar que estas ferramentas são de grande valia para o manejo da cultura do café. Este trabalho foi conduzido na fazenda Brejão, no município de Três Pontas, Minas Gerais, nas safras de 2007/2008, 2008/2009 e 2009/2010, utilizando-se dos atributos químicos do solo, fósforo e potássio, amostrados com o auxílio de um quadriciclo com trado calador e dados de produtividade, que foram obtidos por meio de colheita manual, ambos em pontos georreferenciados. Foi possível caracterizar a magnitude da variabilidade espacial dos atributos em estudo, que apresentaram grande variação no tempo e no espaço. Os melhores ajustes dos semivariogramas permitiram a confecção de mapas mais precisos, contribuindo para a utilização da geoestatística na cafeicultura.
\end{abstract}

PALAVRAS-CHAVE: geoestatística, agricultura de precisão, krigagem, semivariograma, café.

\section{SPATIAL AND TEMPORAL VARIABILITY OF PHOSPHORUS, POTASSIUM AND OF THE YIELD OF A COFFEE FIELD}

\begin{abstract}
The Precision Agriculture appears as an important tool to the management of coffee farms where the knowledge of some soil features associated with the coffee production could help specific application of fertilizing with positive environmental and economic results. So the aim of this study was to use precision agriculture and geoestatistics to evaluate the variables phosphorus, potassium and the coffee plant yield, in three different crops, by evaluating the semivariogram and kriging maps and show that these tools are important to the coffee management. This study was conducted on the Brejão farm in Três Pontas, Minas Gerais, in 2007/2008, 2008/2009 e 2009/2010 crop. As data base were used chemical soil data obtained by sampling in a georreferenced location using a quadricycle with a sampler and a GPS, and the yield data was obtained from manual harvest on the georreferenced location. It was possible to characterize the spatial variability magnitude of the studied attributes, and they presented huge variation on time and space. Adjusts of the best semivariograms enable to produce more accurate maps that contribute to the geostatistics uses on coffee crop.
\end{abstract}

KEYWORDS: geostatistic, precision agriculture, kriging, semivariogram, coffee.

\footnotetext{
${ }^{1}$ Prof. M.Sc., Instituto de Tecnologia, Departamento de Engenharia, Universidade Federal Rural do Rio de Janeiro (UFRRJ), Seropédica - RJ, Doutorando em Engenharia Agrícola, Universidade Federal de Lavras (PPGEA/UFLA), Lavras - MG, gabrielferraz@ufrrj.br.

${ }^{2}$ Prof. Dr., Departamento de Engenharia, Universidade Federal de Lavras (UFLA), Lavras - MG, famsilva@ufla.br.

${ }^{3}$ Eng $^{\mathrm{o}}$ Agrônomo, Mestrando em Engenharia Agrícola, Universidade Federal de Lavras (PPGEA/UFLA), Lavras - MG, lcc.87.ufla@hotmail.com.

${ }^{4}$ Prof. Dr., Faculdade de Agronomia e Medicina Veterinária, Departamento de Solos e Engenharia Rural, Laboratório de Sensoriamento Remoto e Geoinformação, Universidade Federal de Mato Grosso (UFMT), Cuiabá - MT, mdecalves@ufmt.br.

${ }^{5}$ Graduando em Engenharia Agrícola, Departamento de Engenharia, Universidade Federal de Lavras (UFLA,) Lavras - MG, caetanoenagrijr@yahoo.com.br.
}

Recebido pelo Conselho Editorial em: 30-3-2011

Aprovado pelo Conselho Editorial em: 28-10-2011 


\section{INTRODUÇÃO}

O café é um dos produtos mais importantes do agronegócio brasileiro, sendo um grande responsável por receitas cambiais do País, e de acordo com TRABAQUINI et al. (2010), é ainda a base econômica de vários municípios e regiões. De acordo com o Ministério da Agricultura, Pecuária e Abastecimento (BRASIL, 2009), o Brasil é o maior produtor mundial, respondendo por $35,71 \%$ da produção, enquanto o segundo maior produtor, o Vietnã, corresponde a somente $12,42 \%$. Sendo esta cultura tão importante para o Brasil e por envolver elevado custo de produção (OLIVEIRA et al., 2007b; RIBEIRO et al., 2009), o estudo mais aprofundado de novas técnicas que visem ao aumento de produtividade, redução de custos, uso racional de fertilizantes e planejamento eficiente da colheita torna-se de grande interesse.

O cultivo de cafeeiros no Brasil ocorre em ambientes com grande diversidade de clima, solo, relevo, características fisiológicas (cultivares, idade, diâmetro de saia e copa, cor do fruto, etc.), infestações fitossanitárias, manejo (sistema de plantio, sistemas de colheita, sistemas de irrigação, mecanização, sistemas de adubação e pulverização, etc.), dentre muitas outras características. A diversidade desses fatores pode influenciar fortemente sobre a produtividade do cafeeiro e ainda o manejo da lavoura de forma homogênea pode acarretar redução de rentabilidade do produtor rural. Nesse sentido, análises espaciais de produtividade tendem a possibilitar o gerenciamento mais eficiente do processo produtivo (ALVES et al., 2009).

Outro fator muito importante de ser analisado é o solo. Na atividade cafeeira, ainda hoje, muitos produtores não realizam análises do solo com a frequência necessária, desconhecendo os parâmetros mínimos para o manejo da fertilidade do solo de forma racional e eficiente. De acordo com SILVA et al. (2003), o estudo da variabilidade espacial de propriedades do solo tem grande importância na escolha de uma área experimental, na coleta de amostras e na interpretação de resultados, no levantamento e classificação de solos e nos esquemas de uso racional de fertilizantes.

De acordo com BLISKA et al. (2009), nos últimos anos, o agronegócio do café brasileiro internalizou novas técnicas de produção que promoveram impactos positivos sobre produtividade, competitividade e qualidade final do produto.

Segundo OLIVEIRA et al. (2007 a), SILVA et al. (2008) e CARVALHO et al. (2009), a agricultura de precisão apresenta-se como um conjunto de tecnologias capaz de auxiliar o produtor rural a identificar as estratégias a serem adotadas para aumentar a eficiência no gerenciamento da agricultura. Inclui maximizar a rentabilidade da produção, tornando o agronegócio mais competitivo face ao processo de globalização da economia, e contribuir, assim, para o sucesso da atividade agrícola.

A agricultura de precisão na cafeicultura vem sendo denominada de Cafeicultura de Precisão, que pode ser definida como o conjunto de técnicas e tecnologias capaz de auxiliar o cafeicultor a manejar sua lavoura, baseando-se na variabilidade espacial dos atributos do solo e da planta, visando a maximizar a rentabilidade, a aumentar eficiência da adubação, pulverização e colheita, culminando na elevação da produtividade e da qualidade final do produto.

A geoestatística é uma ferramenta importante para análise de dados no âmbito da agricultura de precisão. Segundo VIEIRA (2000), o objetivo da geoestatística aplicada à agricultura de precisão é caracterizar a magnitude da variabilidade espacial dos atributos do solo e das plantas, e fazer estimativa, utilizando o princípio da variabilidade espacial a fim de se identificarem inter-relações desses atributos no espaço e no tempo, além de permitir estudar padrões de amostragem adequada. Desta forma, o uso dessa técnica na cafeicultura de precisão ganha destaque, pois pode contribuir com o produtor de café na identificação da variabilidade espacial dos fatores produtivos, possibilitando o melhor gerenciamento de sua lavoura.

Este estudo teve como objetivo utilizar técnicas da cafeicultura de precisão aliadas às ferramentas da geoestatística, para caracterizar a magnitude da variabilidade espacial do fósforo, potássio e da produtividade de uma lavoura cafeeira, utilizando ajustes de semivariogramas a partir 
de diferentes métodos e modelos, de forma a encontrar aqueles que melhor caracterizem os atributos e desta maneira possibilitem a confecção de mapas de isolinhas interpolados por krigagem, demonstrando quão valiosa esta técnica é para o manejo da cultura do cafeeiro.

\section{MATERIAL E MÉTODOS}

O experimento foi desenvolvido na fazenda Brejão, localizada no município de Três Pontas, sul de Minas Gerais, em uma área de 22 ha de lavoura de cafeeiro (Coffea arabica L.) da cultivar Topázio, plantada em dezembro de 2005, no espaçamento de $3,8 \mathrm{~m}$ entre linhas e $0,8 \mathrm{~m}$ entre plantas, totalizando 3.289 plantas ha $^{-1}$. As coordenadas geográficas do ponto central da área são de $21^{\circ} 25^{\prime} 58^{\prime \prime}$ de latitude sul e $45^{\circ} 24^{\prime} 51^{\prime \prime}$ de longitude oeste de Greenwich. Os pontos limites da área foram obtidos por meio do uso de GPS geodésico (precisão de $\pm 10 \mathrm{~cm}$ ).

O clima do local é caracterizado como ameno, tropical de altitude, com temperaturas moderadas, verão quente e chuvoso, classificado por Köeppen como Cwa. O solo da área foi classificado como Latossolo Vermelho-Amarelo.

Para a coleta dos dados de produtividade, demarcou-se na área uma malha irregular, num total

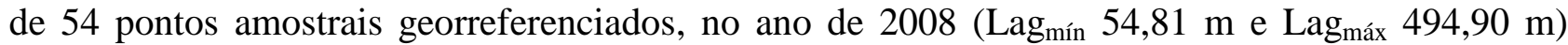
(Figura 1A), e uma malha irregular com 48 pontos nos anos de 2009 e 2010 ( $\operatorname{Lag}_{\text {mín }} 24,12 \mathrm{~m}$ e Lag $_{\text {máx }}$ 537,82 m) (Figura 1B). Para a demarcação desses pontos, foi utilizado um GPS de navegação (precisão de $\pm 5 \mathrm{~m}$ ). Cada ponto amostral corresponde a quatro plantas: duas plantas localizadas na rua de cafeeiros onde o ponto foi georreferenciado, e as outras duas plantas localizadas em cada rua lateral ao ponto de referencia.

A produtividade de café (L planta ${ }^{-1}$ ) foi obtida por meio da colheita manual sobre panos das quatro plantas em torno do ponto amostral, e o volume colhido de cada planta, após a abanação, foi medido em um recipiente graduado em litros. Após essa medição, foi obtida a média de produtividade destas quatro plantas, resultando no valor de produtividade para o ponto amostral.

Para a coleta dos dados de fertilidade do solo, demarcou-se na área uma malha de um ponto por hectare, num total de 22 pontos amostrais georreferenciados (Figuras 1C e 1D). Para o ano de 2007, o Lag $_{\text {mín }}$ foi de 54,81 m e o Lag máx foi de 503,98 m (Figura 1C), e para os anos de 2008 e 2009 o Lag $_{\text {mín }}$ foi de 83,62 m e o Lagmáx foi de 508,28 m (Figura 1D). Para a demarcação dos pontos de coleta e para a amostragem de solo, utilizou-se um quadriciclo com broca pneumática e GPS geodésico. Em cada hectare, foi realizado um caminhamento aleatório para a coleta de 8 subamostras que foram homogeneizadas para gerar 1 amostra composta. Tais subamostras foram retiradas na projeção da saia do cafeeiro de 0 a $20 \mathrm{~cm}$ de profundidade, no mês de julho dos anos de 2007, 2008 e 2009. Essa metodologia corresponde àquela que vem sendo utilizada por empresas prestadoras de serviços de mapeamento da variabilidade dos atributos do solo para a cultura do café.
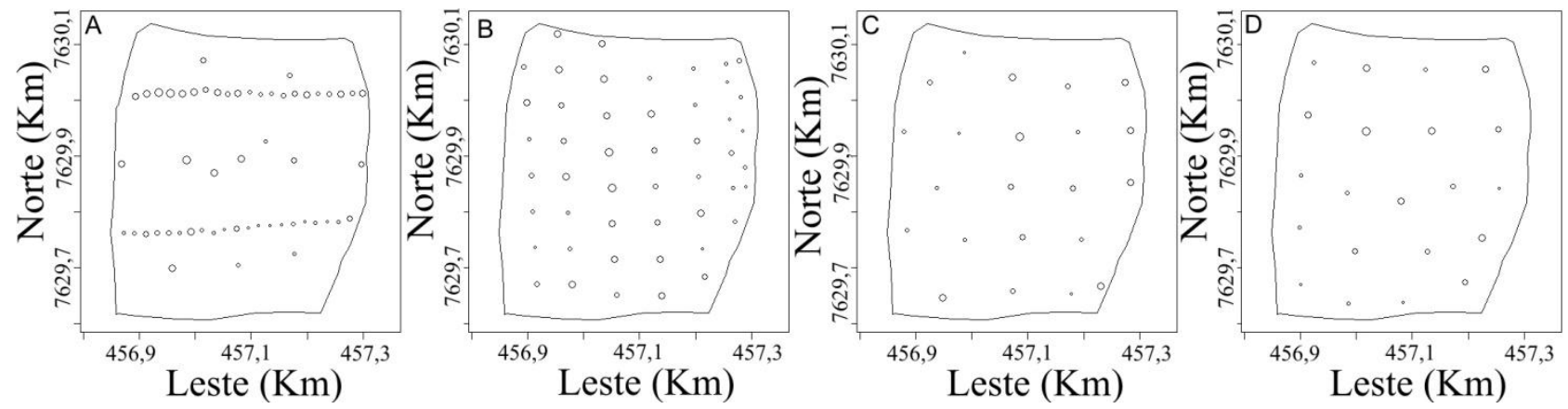

FIGURA 1. Grade de pontos amostrais georreferenciados para a coleta dos dados de produtividade nos anos de 2008 (A) e 2009 e 2010 (B) e coleta de solo em 2007 (C) e em 2008 e 2009 (D). Sampling points grid to collect yield data in 2008 (A) and 2009 and 2010 (B) and to collect soil data in 2007 (C) and in 2008 and 2009 (D). 
As amostras de solo foram enviadas ao Laboratório de Análise de Solo e Folha da Cooperativa dos Cafeicultores da Zona de Três Pontas (COCATREL) para se procederem às análises dos atributos Fósforo (P) pelo método Mehlich-1 e Potássio (K) pelo método Mehlich-1.

A dependência espacial do fósforo, do potássio e da produtividade de café foi analisada por meio de ajustes de semivariogramas, clássico e robusto, e interpolação por krigagem ordinária. O semivariograma clássico foi estimado como segue:

$$
\hat{\gamma}(\mathrm{h})=\frac{1}{2 \mathrm{~N}(\mathrm{~h})} \sum_{\mathrm{i}=1}^{\mathrm{N}(\mathrm{h})}\left[\mathrm{Z}\left(\mathrm{x}_{\mathrm{i}}\right)-\mathrm{Z}\left(\mathrm{x}_{\mathrm{i}}+\mathrm{h}\right)\right]^{2}
$$

em que, $N(h)$ é o número de pares experimentais de observações $Z\left(x_{i}\right)$ e $Z\left(x_{i}+h\right)$ separados por uma distância h. O semivariograma é representado pelo gráfico $\hat{\gamma}(h)$ versus h. Do ajuste de um modelo matemático aos valores calculados de $\hat{\gamma}(h)$, são estimados os coeficientes do modelo teórico para o semivariograma denominado de efeito pepita, $\mathrm{C}_{0}$; patamar, $\mathrm{C}_{0}+\mathrm{C}_{1}$; e o alcance, a.

De acordo com WEBSTER \& OLIVER (2007), o estimador robusto dos valores do semivariograma é menos suscetível à influência dos valores da massa de dados do que o estimador clássico. Assim, o estimador robusto é descrito por:

$$
\hat{\gamma}(\mathrm{h})=\frac{1}{2} \frac{\left[\mathrm{N}(\mathrm{h})^{-1} \sum_{\mathrm{N}(\mathrm{h})} \sqrt{\mid(\mathrm{Z}(\mathrm{s}+\mathrm{h})-\mathrm{Z}(\mathrm{s}))}\right]^{4}}{0,457+\frac{0,494}{\mathrm{~N}(\mathrm{~h})}}
$$

Este estimador assume que as diferenças $\mathrm{Z}(\mathrm{s}+\mathrm{h})-\mathrm{Z}(\mathrm{s})$ são distribuídas de forma normal para todos os pares $(\mathrm{s}+\mathrm{h}, \mathrm{s})$. A transformação da raiz quadrada das diferenças é apresentada como tendo momentos parecidos com aqueles da distribuição normal, e o denominador da equação é a correção de tendências.

O ajuste dos modelos de semivariogramas foi escolhido em função dos Mínimos Quadrados Ordinários (OLS - ordinary least square), Mínimos Quadrados Ponderados (WLS - weighted least squares), estimados pelo modo clássico e pelo modo robusto, e a Máxima Verossimilhança (ML maximum likelihood) e a Máxima Verossimilhança Restrita (REML - restricted maximum likelihood), usando o estimador clássico. Foram testados, para todos os métodos, os modelos esférico, exponencial e o gaussiano, o que totalizou 18 semivariogramas para cada variável. Para a escolha dos métodos e dos modelos de ajuste do semivariograma, foi levada em consideração a validação cruzada dos dados (FARACO et al., 2008; JOHANN et al., 2010). Após o ajuste dos semivariogramas foi realizada a interpolação dos dados por krigagem ordinária de forma a possibilitar a visualização dos padrões de distribuição espacial das variáveis na lavoura.

De acordo com ISAAKS \& SRIVASTAVA (1989), a validação cruzada é a técnica de avaliação de erros de estimativa que permite comparar valores preditos com os amostrados. O valor da amostra, em certa localização $\mathrm{Z}\left(\mathrm{s}_{\mathrm{i}}\right)$, é temporariamente descartado do conjunto de dados e, então, é feita uma previsão por krigagem no local $\hat{Z}\left(s_{(i)}\right)$, usando-se as amostras restantes. Desta forma, é possível retirar alguns valores que serão muito úteis para a escolha do método como o Erro Médio (EM), o Desvio-Padrão dos Erros Médios (DP $\mathrm{EM}_{\mathrm{EM}}$, o Erro Médio Reduzido (ER), e o Desvio-Padrão dos Erros Médios Reduzidos (DP $\mathrm{ER}_{\mathrm{ER}}$. Assim, o Erro Médio por validação cruzada (EM) é obtido pela seguinte expressão:

$$
\mathrm{EM}=\frac{1}{\mathrm{n}} \sum_{\mathrm{i}=1}^{1}\left(\mathrm{Z}\left(\mathrm{s}_{\mathrm{i}}\right)-\hat{\mathrm{Z}}\left(\mathrm{s}_{(\mathrm{i})}\right)\right)
$$

em que, $n$ é o número de dados; $\mathrm{Z}\left(\mathrm{s}_{\mathrm{i}}\right)$ é valor observado no ponto $s_{i}$, e $\hat{Z}\left(\mathrm{~s}_{(\mathrm{i})}\right)$ o valor predito por krigagem ordinária no ponto $s_{i}$, sem considerar a observação $Z\left(\mathrm{~s}_{\mathrm{i}}\right)$ (FARACO et al., 2008). 
O erro médio reduzido (ER) é definido pela equação:

$$
\mathrm{ER}=\frac{1}{\mathrm{n}} \sum_{\mathrm{i}=1}^{\mathrm{n}} \frac{\mathrm{Z}\left(\mathrm{s}_{\mathrm{i}}\right)-\hat{\mathrm{Z}}\left(\mathrm{s}_{(\mathrm{i})}\right)}{\sigma\left(\hat{\mathrm{Z}}\left(\mathrm{s}_{(\mathrm{i})}\right)\right)}
$$

em que, $\sigma\left(\hat{Z}\left(s_{(i)}\right)\right)$ é o desvio-padrão da krigagem no ponto $s_{i}$, sem considerar a observação $Z\left(s_{i}\right)$.

$\mathrm{O}$ desvio-padrão dos erros reduzidos $\left(\mathrm{S}_{\mathrm{ER}}\right)$ é obtido a partir de:

$$
S_{E R}=\sqrt{\frac{1}{n} \sum_{\mathrm{i}=1}^{\mathrm{n}}\left\{\frac{\mathrm{Z}\left(\mathrm{s}_{\mathrm{i}}\right)-\hat{\mathrm{Z}}\left(\mathrm{s}_{(\mathrm{i})}\right)}{\sigma\left(\hat{\mathrm{Z}}\left(\mathrm{s}_{(\mathrm{i})}\right)\right)}\right\}^{2}}
$$

A diferença média entre os valores será mais próxima de zero quanto melhor for a estimativa. Os critérios de escolha baseados na validação cruzada devem encontrar o valor de EM e de ER mais próximo de zero, o valor $\mathrm{DP}_{\mathrm{EM}}$ deve ser o menor, e o valor de $\mathrm{S}_{\mathrm{ER}}$ deve ser o mais próximo de um.

Para as análises estatísticas e geoestatísticas e na plotagem dos mapas, foi utilizado sistema computacional estatístico R Development Core Team, por meio da biblioteca geoR (RIBEIRO JUNIOR \& DIGGLE, 2001). Os mapas foram gerados na coordenada Universal Transversa de Mercator (UTM) na zona 23K, na qual está inserida a região de Três Pontas.

\section{RESULTADOS E DISCUSSÃO}

Pela análise da Tabela 1, nenhuma variável estudada apresentou coeficiente de variação (C.V.) menor que $10 \%$, indicando que todas possuíam variabilidade, pois segundo GOMES \& GARCIA (2002), C.V. menor que $10 \%$ indicaria homogeneidade, e de acordo com FROGBROOK et al. (2002), os valores elevados de C.V. podem ser considerados como os primeiros indicadores da existência de heterogeneidade nos dados. Na Tabela 1, observa-se ainda que o fósforo (P), em todos os anos, apresentou os maiores valores de CV. SILVA et al. (2008), em seus estudos, também encontraram valores de C.V. altos para o $\mathrm{P}$.

Os coeficientes de Assimetria e de Curtose são utilizados para se observar a distribuição estatística das variáveis (MAPA \& KUMARAGAMAGE, 1996). Os valores de assimetria e curtose devem ser próximos de zero, embora alguns softwares utilizam valores de curtose próximos de 3 (SILVA et al., 2008). De acordo com KERRY \& OLIVER (2007), quando os valores de assimetria estão entre -1 e 1, não é necessário transformar os dados antes de calcular os semivariogramas. Para valores de assimetria superiores a 1, WEBSTER (2001) cita que é necessário realizar uma transformação logarítmica dos dados, para que, desta forma, estes possam apresentar distribuição normal. Sendo assim, foi necessário realizar a transformação logarítmica das variáveis P07, P08, P09 e K09 (Tabela 1).

TABELA 1. Estatística descritiva para as variáveis potássio $\left(\mathrm{mg} \mathrm{dm}^{-3}\right)$ e fósforo $\left(\mathrm{mg} \mathrm{dm}^{-3}\right)$, nos anos de 2007, 2008 e 2009, e da produtividade do cafeeiro (L planta $\left.{ }^{-1}\right)$, em 2008, 2009 e 2010. Descriptive statistics to the variables potassium $\left(\mathrm{mg} \mathrm{dm}^{-3}\right)$ and phosphorus $\left(\mathrm{mg} \mathrm{dm}^{-3}\right)$ in 2007, 2008 and 2009, and coffee yield $\left(L\right.$ plant $\left.^{-1}\right)$ in 2008, 2009 and 2010.

\begin{tabular}{cccccrrrrr}
\hline & Mín. & \multicolumn{1}{c}{ Máx. } & Média & Mediana & \multicolumn{1}{c}{ DP } & \multicolumn{1}{c}{ Var } & \multicolumn{1}{c}{ C.V. } & \multicolumn{1}{c}{ k } & Assimetria \\
\hline K 07 & 143 & 226,00 & 185,20 & 186 & 24,69 & 609,68 & 13,33 & $-0,90$ & 0,06 \\
K 08 & 88 & 207,00 & 150,50 & 150 & 38,66 & $1.494,76$ & 25,69 & $-1,46$ & $-0,04$ \\
K 09 & 88 & 174,00 & 116,90 & 113 & 24,05 & 578,53 & 20,58 & 1,38 & 1,08 \\
P 07 & 3 & 64,00 & 16,14 & 8,00 & 17,49 & 305,93 & 108,39 & 3,51 & 1,99 \\
P 08 & 1 & 35,00 & 10,00 & 7,00 & 9,13 & 83,40 & 91,32 & 2,38 & 1,75 \\
P 09 & 3 & 66,00 & 9,19 & 5,00 & 13,49 & 182,06 & 146,82 & 17,74 & 4,10 \\
Prod 08 & 0,03 & 3,95 & 1,45 & 1,21 & 0,96 & 0,92 & 65,98 & $-0,64$ & 0,44 \\
Prod 09 & 0,68 & 6,49 & 2,72 & 2,46 & 1,36 & 1,85 & 50,01 & 0,31 & 0,83 \\
Prod 10 & 1,08 & 10,2 & 4,93 & 4,59 & 2,09 & 4,41 & 42,57 & $-0,29$ & 0,45 \\
\hline \multicolumn{7}{l}{ Mín - valor mínimo da variável; Máx - Valor máximo da variável; DP - Desvio-Padrão; Var - Variância; C.V. - Coeficiente de }
\end{tabular}


A partir da análise dos valores mínimos e máximos, e também da média dos atributos, tanto do solo quanto da planta, é possível perceber que existe grande variação nos dados. Porém, somente o conhecimento dessa amplitude não é suficiente para identificar os locais onde se encontram os altos teores e os locais onde se encontram os teores mais baixos de uma determinada variável. Neste caso, torna-se é necessário lançar mão de ferramentas geoestatísticas, para que se possa identificar a variabilidade espacial dos dados, bem como para realizar a confecção de mapa, a fim de possibilitar o gerenciamento preciso das intervenções necessárias.

Todas as variáveis foram submetidas aos critérios da validação cruzada, o que possibilitou a escolha do método de ajuste do modelo de semivariograma que melhor se adequasse a cada uma (Tabela 2). Sendo assim, a variável fósforo teve seu ajuste segundo o método dos Mínimos Quadrados Ponderados, utilizando o estimador clássico (WLS-C) para o ano de 2007 e da Máxima Verossimilhança (ML) para os anos de 2008 e 2009; o potássio ajustou-se segundo o método dos Mínimos Quadrados Ordinários, utilizando o estimador robusto (OLS-R) para os anos de 2007 e 2008, e para o ano de 2009 o ajuste do semivariograma deu-se em função da Máxima Verossimilhança Restrita (REML), que foi o método escolhido também para a produtividade em 2008 e 2010. Já o semivariograma da produtividade, do ano de 2009, foi ajustado pela Máxima Verossimilhança (ML) (Tabela 2 e Figura 2).

TABELA 2. Métodos, modelos e parâmetros estimados dos semivariogramas experimentais para as variáveis: potássio $\left(\mathrm{mg} \mathrm{dm}^{-3}\right)$ e fósforo $\left(\mathrm{mg} \mathrm{dm}^{-3}\right)$, nos anos de 2007, 2008 e 2009, e para a produtividade da cultura do cafeeiro $\left(\mathrm{L}\right.$ planta $\left.^{-1}\right)$, nos anos de 2008, 2009 e 2010. Methods, models and parameters estimates by the experimental variogram to the variables: potassium $\left(\mathrm{mg} \mathrm{dm}^{-3}\right)$ and phosphorus $\left(\mathrm{mg} \mathrm{dm}^{-3}\right)$ in 2007,2008 and 2009 and to coffee yield $\left(\mathrm{L}\right.$ plant $\left.{ }^{-1}\right)$ in 2008, 2009 and 2010.

\begin{tabular}{cccccccccccc}
\hline & Método & Modelo & $\mathbf{C}_{\mathbf{0}}$ & $\mathbf{C}_{\mathbf{1}}$ & $\mathbf{C}_{\mathbf{0}}+\mathbf{C}_{\mathbf{1}}$ & $\mathbf{a}$ & $\mathbf{a}$ & $\mathbf{E M}$ & $\mathbf{D P}_{\mathbf{E M}}$ & $\mathbf{E R}$ & $\mathbf{S}_{\mathbf{E R}}$ \\
\hline P 07 & WLS -C & Esf & 0,00 & 0.148 & 0,148 & 133,31 & 133,31 & 0,00202 & 0,419 & 0,002561 & 1,104 \\
P 08 & ML & Esf & 0,00 & 0,147 & 0,147 & 89,73 & 89,73 & 0,0000 & 0,000 & $-0,000007$ & 1,050 \\
P 09 & ML & Exp & 0,00 & 0,091 & 0,091 & 52,33 & 156,76 & 0,00012 & 0,3069 & 0,000208 & 1,031 \\
K 07 & OLS - R & Exp & 0 & 717,45 & 717,45 & 55,16 & 165,25 & 0,01782 & 23,953 & 0,00036 & 0,925 \\
K 08 & OLS - R & Esf & 0 & $2.486,88$ & $2.486,88$ & 437,67 & 437,67 & $-0,7694$ & 36,017 & $-0,01372$ & 1,279 \\
K 09 & REML & Esf & 0,0018 & 0,0054 & 0,0072 & 83,7 & 83,7 & 0,000 & 0,000 & 0,0891 & 1,024 \\
Prod 08 & REML & Esf & 0,26 & 0,63 & 0,89 & 217,24 & 217,24 & 0,02571 & 0,704 & 0,01743 & 1,012 \\
Prod 09 & ML & Esf & 0,63 & 1,26 & 1,90 & 280,51 & 280,51 & 0,00553 & 1,093 & 0,00260 & 1,017 \\
Prod 10 & REML & Esf & 2,14 & 2,54 & 4,68 & 203,41 & 203,41 & 0,00699 & 1,935 & 0,00183 & 1,013 \\
\hline
\end{tabular}

$\mathrm{C}_{0}$ - Efeito Pepita; $\mathrm{C}_{1}$ - Contribuição; $\mathrm{C}_{0}+\mathrm{C}_{1}$ - Patamar; a - alcance; a' - alcance prático; EM - Erro Médio; $\mathrm{DP}_{\mathrm{EM}}-\mathrm{Desvio}_{\mathrm{P}}$ Padrão do Erro Médio; ER - Erro Médio Reduzido; $\mathrm{S}_{\mathrm{ER}}$ - Desvio-Padrão dos Erros Médios Reduzidos; WLS - C - Mínimos Quadrados Ponderados estimador Clássico; ML - Máxima Verossimilhança; OLS - R - Mínimos Quadrados Ordinário estimador Robusto; REML - Máxima Verossimilhança Restrita; Esf - Esférico; Exp - Exponencial.

Baseando-se na metodologia de análise geoestatística, foi possível quantificar a magnitude e a estrutura de dependência espacial de todas as variáveis e em todos os anos estudados, o que pode ser observado na Figura 2. 

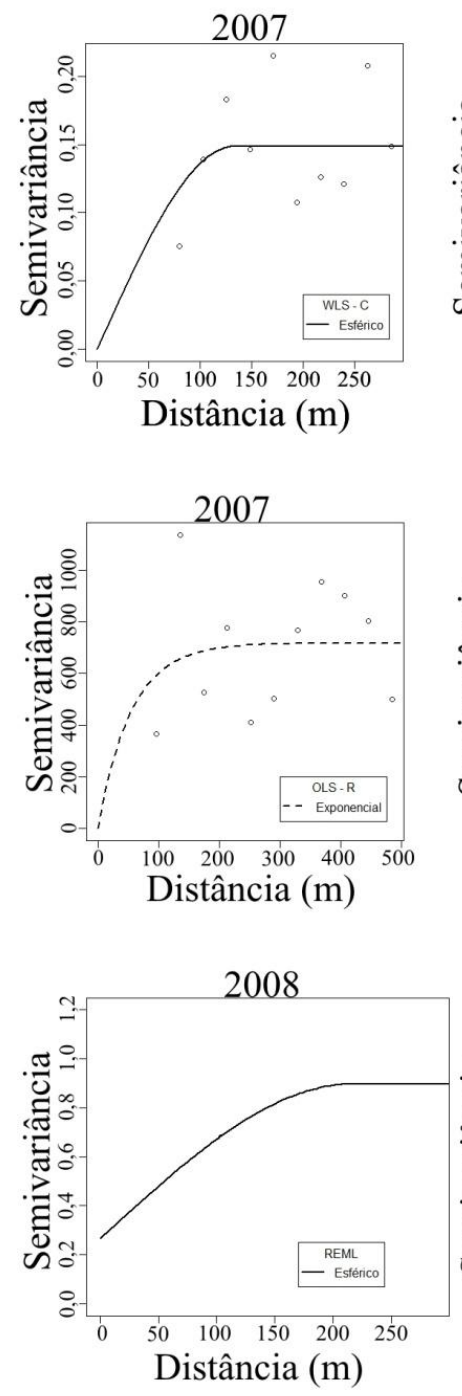

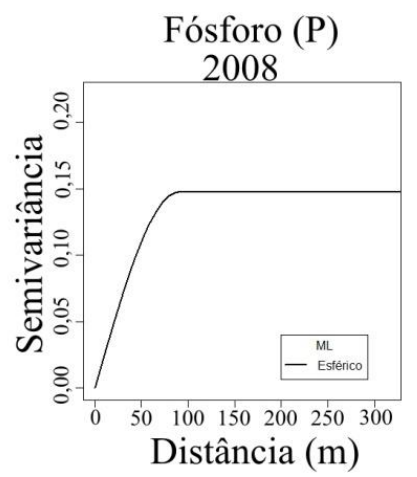

Potássio $(\mathrm{K})$

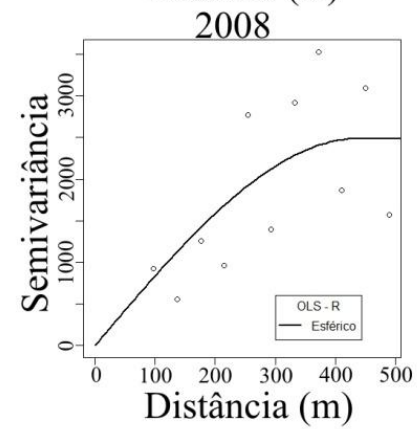

Produtividade

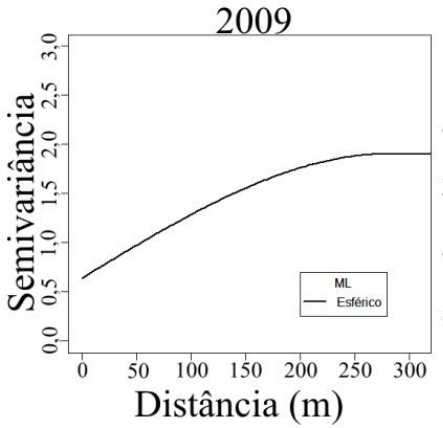

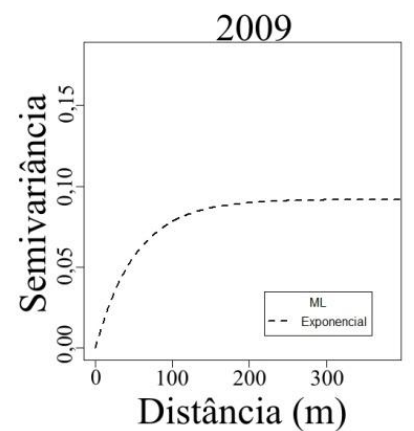
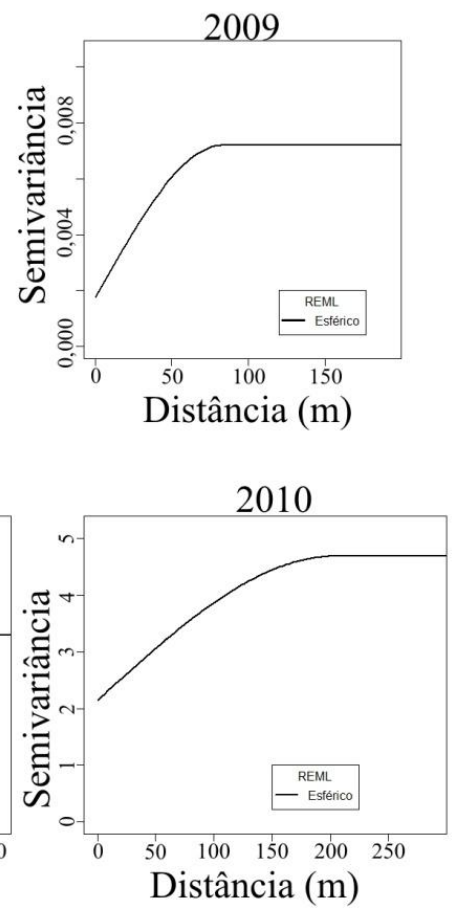

FIGURA 2. Semivariogramas escolhidos em função do método e modelo para as variáveis químicas do solo: fósforo $\left(\mathrm{mg} \mathrm{dm}^{-3}\right)$ e potássio $\left(\mathrm{mg} \mathrm{dm}^{-3}\right)$ nos anos de 2007, 2008 e 2009, e da produtividade $\left(\mathrm{L} \mathrm{planta}^{-1}\right)$ em 2008, 2009 e 2010. Semivariograms chosen based on the methods and models to the soil chemicals variables: phosphorus $\left(\mathrm{mg} \mathrm{dm}^{-3}\right)$ and potassium $\left(\mathrm{mg} \mathrm{dm}^{-3}\right)$ in 2007, 2008 and 2009, and to coffee yield $\left(\mathrm{L}\right.$ plant $\left.{ }^{-1}\right)$ in 2008, 2009 and 2010.

Segundo WEBSTER \& OLIVER (2007), o modelo matemático esférico é o mais frequentemente utilizado em geoestatística, porém o modelo exponencial também é muito utilizado. SILVA et al. (2007) e SILVA et al. (2008), realizando estudos em uma lavoura cafeeira, encontraram que o modelo esférico se ajustou tanto para as variáveis fósforo e potássio quanto para a produtividade. SILVA et al. (2010), em seus estudos de uma lavoura cafeeira em três safras, ajustaram o modelo esférico para a produtividade. Neste trabalho, o modelo esférico foi o que mais se ajustou às variáveis (P07, P08, K08, K09, Prod 08, 09 e 10), e o modelo exponencial foi ajustado a duas variáveis (P09, K07).

O efeito pepita é um importante parâmetro do semivariograma, e indica variabilidade não explicada, considerando a distância de amostragem utilizada. Percebeu-se que o efeito pepita zero foi encontrado para a variável P 07, P 08 e P 09 K 07 e K 08.

Os valores do alcance dos semivariogramas têm uma importância considerável na determinação do limite da dependência espacial, o que pode ser, também, um indicativo do intervalo entre unidades de mapeamento de solos ou, ainda, dos atributos relacionados às plantas. 
Para o modelo esférico, o alcance é dado por a, já para o modelo exponencial usa-se o alcance prático a'. Isto porque este modelo não possui um alcance finito; assim, para fins práticos, é conveniente utilizar um alcance prático que corresponde a aproximadamente três vezes o valor de a (WEBSTER \& OLIVER 2007).

As variáveis estudadas apresentaram diferentes alcances de dependência espacial: a variável P apresentou valores de alcance de 133,31 m, em 2007, 89,73 m, em 2008, e 156,76 m, em 2009. A variável K apresentou valores de 165,25 m (2007), 437,67 m (2008) e 83,7 m (2009). Essas variações de alcance ocorridas entre os anos podem ser em função do manejo baseado em agricultura de precisão realizado na área. Desta maneira, o planejamento de uma amostragem de solo na área, que contemplasse a variabilidade espacial existente, teria de observar estas diferenças de alcance existentes nos dois atributos estudados, utilizando-se sempre de valores menores que o menor valor de alcance encontrado para se construir a grade de amostragem mais adequada. A produtividade apresentou valores de alcance iguais a 217,24 m (2008), 280,51 m (2009) e 203,41 m (2010), o que demonstra que a produtividade dessa lavoura apresenta dependência espacial até valores superiores a $200 \mathrm{~m}$.

SILVA et al. (2008), estudando duas safras de uma lavoura cafeeira (2002/2003 e 2003/2004), encontraram que a variável $\mathrm{P}$ apresentou efeito pepita de 0,09 e um alcance de $86,05 \mathrm{~m}$ na primeira safra e 0,52 e $210,04 \mathrm{~m}$, respectivamente, na segunda safra. Para o K, os valores da primeira safra foram 579 e 142,08 m, e na segunda safra " $\mathrm{C}_{0}$ " igual a 973 e "a" igual a $188,02 \mathrm{~m}$. Para a produtividade, os autores encontraram os valores de efeito pepita iguais a 0,58 e 0,79, e alcance de $65,04 \mathrm{~m} \mathrm{e} 60,43 \mathrm{~m}$, respectivamente, para a primeira e segunda safras. SILVA et al. (2010), estudando a produtividade de cafeeiros em três safras, encontraram valores de alcance iguais a $21,3 \mathrm{~m}, 27,6 \mathrm{~m}, 36,0 \mathrm{~m}$ e efeito pepita igual a 0 , para as duas primeiras safras, e 0,76 na última safra estudada. Essas diferenças encontradas entre os autores e o presente trabalho podem ser devidas à idade das plantas, à cultivar, ao relevo da lavoura e aos tratamentos fitossanitários realizados em cada área estudada.

Posteriormente, foram estimados valores da produtividade, do fósforo e do potássio, pela krigagem ordinária, tendo como base a dependência espacial dos modelos de semivariogramas. Sendo assim, foi possível construir mapas de distribuição espacial para todas as variáveis deste estudo (Figura 3), que permitiram visualizar a variabilidade espacial na área em cada ano, além de possibilitar a observação da variabilidade temporal.

De acordo com PROCAFÉ (2011), o nível nutricional de fósforo (P) é considerado baixo quando a amostragem de solo indicar valores menores que $10 \mathrm{mg} \mathrm{dm}^{-3}$, médio para valores entre 10 e $20 \mathrm{mg} \mathrm{dm}^{-3}$ e alto para valores superiores a $20 \mathrm{mg} \mathrm{dm}^{-3}$. Pela análise dos mapas de fósforo, podese perceber que, no ano de 2007 , a região oeste e nordeste apresentaram valores nutricionais baixos, enquanto a região norte e a região sudeste do mapa apresentaram valores nutricionais altos. Para o ano de 2008, as partes central, sul e sudeste apresentaram valores nutricionais altos, enquanto a parte nordeste apresentou valores nutricionais baixos. No ano de 2009, valores nutricionais baixos ocuparam grande fatia do mapa, sendo que os valores nutricionais considerados altos foram apresentados em apenas um ponto do mapa.

PROCAFÉ (2011) estabeleceu que o nível nutricional de potássio (K) é considerado baixo quando a amostragem de solo indicar valores menores que $100 \mathrm{mg} \mathrm{dm}^{-3}$, médio para valores entre 100 e $160 \mathrm{mg} \mathrm{dm}^{-3}$ e alto para valores superiores a $160 \mathrm{mg} \mathrm{dm}^{-3}$. Observando o mapa de potássio no ano de 2007, pode-se perceber que havia poucos locais onde estava abaixo de $160 \mathrm{mg} \mathrm{dm}^{-3}$, localizando-se na região oeste do mapa. Já no ano de 2008, as regiões nordeste e oeste apresentaram valores considerados baixos, e grande parte do mapa apresentou valores médios, sendo que as partes leste, sudeste e nordeste apresentaram valores altos. No ano de 2009, perceberam-se poucos pontos com valores nutricionais altos, sendo a predominância de valores médios.

Observando-se os mapas de fósforo e potássio, ano a ano, percebem-se, facilmente, as localidades em que era necessária a aplicação de fertilizantes, e os locais onde ela não se fazia 
necessária, evidenciando o problema de se usar o valor médio para recomendação de adubação do solo. Portanto, a utilização destes mapas contribuiu para o manejo diferenciado da lavoura, permitindo assim aplicações mais precisas e eficientes de fertilizantes. Esses mapas possibilitaram, ainda, identificar como esses atributos variaram ao longo dos três anos em estudo.

A produtividade média encontrada na colheita de 2008 foi de $1,45 \mathrm{~L}_{\text {planta }}{ }^{-1}, 2,72 \mathrm{~L}_{\text {planta }}{ }^{-1}$ em 2009, e para o ano de 2010, a produtividade média dessa lavoura foi de 4,93 $\mathrm{L} \mathrm{planta}^{-1}$. Ao se analisar o mapa de produtividade dos anos de 2008 e 2009, pôde-se perceber que a região central destes mapas representa as maiores produtividades da lavoura, enquanto na parte mais oeste do mapa, a produtividade é menor. No ano de 2010, o local de maior produtividade foi a região sul da área, e os locais de baixa produtividade ficaram dispersos na área. Ressalta-se que a porção central, norte e sul se encontram mais elevadas que as demais porções.
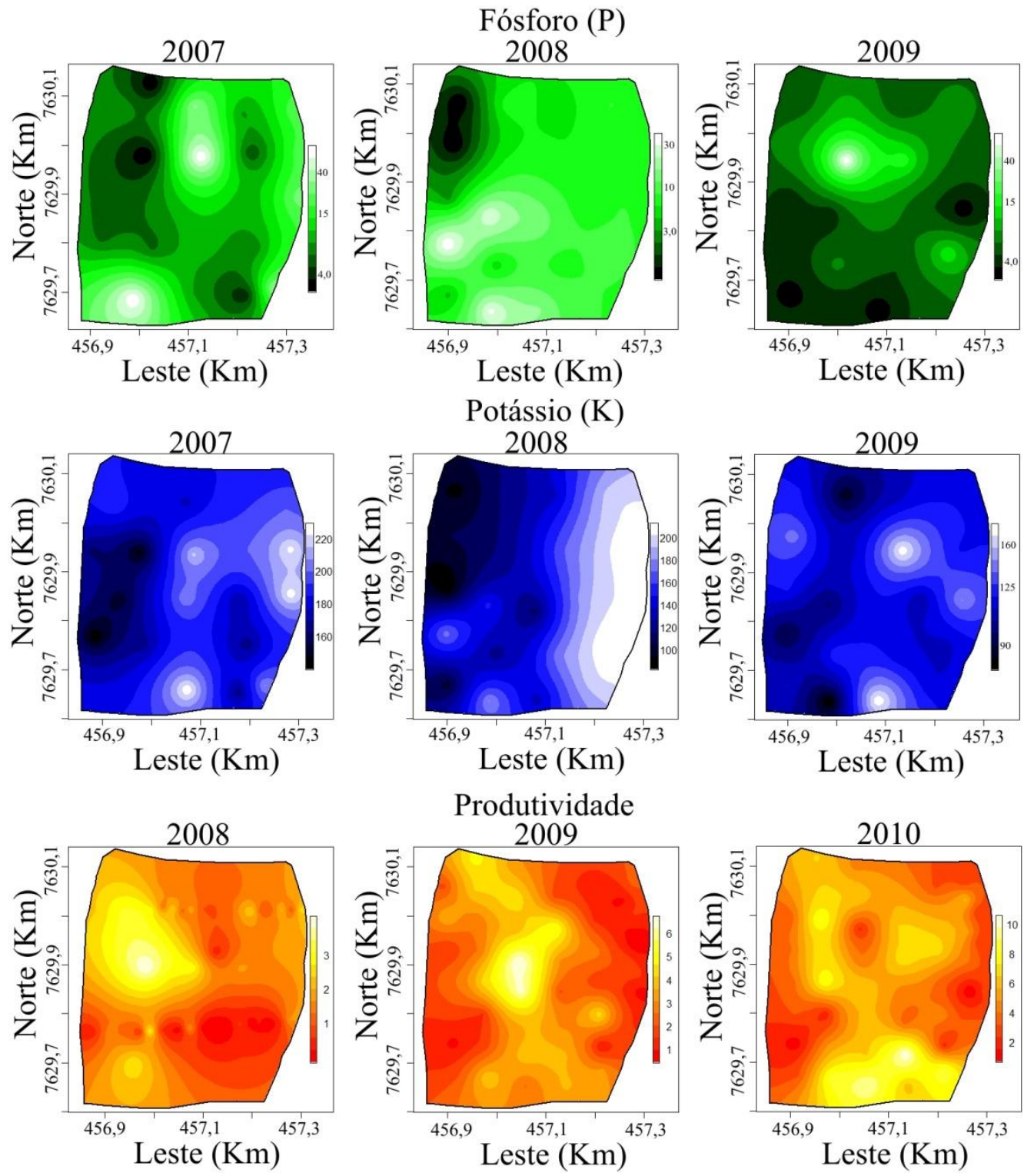

FIGURA 3. Distribuição espacial do fósforo $\left(\mathrm{mg} \mathrm{dm}^{-3}\right)$ e do potássio $\left(\mathrm{mg} \mathrm{dm}^{-3}\right) \mathrm{em} \mathrm{2007,} 2008 \mathrm{e}$ 2009 e da produtividade ( L planta $^{-1}$ ) nos anos de 2008, 2009 e 2010. Spatial distribution of phosphorus $\left(\mathrm{mg} \mathrm{dm}^{-3}\right)$ and potassium $\left(\mathrm{mg} \mathrm{dm}^{-3}\right)$ in 2007,2008 and 2009 and coffee yield $\left(\mathrm{L}\right.$ plant $\left.^{-1}\right)$ in 2008, 2009 and 2010.

Desta forma, a utilização de mapas deve vir em conjunto com a manipulação dos mapas de atributos químicos do solo, objetivando-se a encontrar os motivos da ocorrência da variação da 
produtividade, principalmente em se tratando de baixas produtividades. Os mapas de produtividade, obtidos pela metodologia utilizada neste trabalho, podem ainda ser utilizados no gerenciamento da colheita, tanto manual quanto mecanizada ou semimecanizada. Na colheita manual, esses mapas contribuirão na estimativa de produtividade e na contratação de pessoal para a colheita. Na colheita semimecanizada, serão de suma importância, além dos citados para a colheita manual, na aquisição ou aluguel de derriçadoras portáteis. Na colheita mecanizada do café, podem ser utilizados para se planejar o esvaziamento da carreta do trator de apoio à colhedora ou em caso de colhedoras que possuem reservatórios próprios, no planejamento do momento de se esvaziarem os mesmos, o que poder reduzir paradas e manobras desnecessárias.

\section{CONCLUSÕES}

Os semivariogramas permitiram a caracterização da magnitude da variabilidade espacial dos atributos químicos do solo, fósforo e potássio, e também da produtividade da cultura do cafeeiro. O teste de diferentes métodos e modelos permitiu identificar aquele que melhor se ajustou a cada uma das variáveis. A interpolação por krigagem permitiu a confecção de mapas de isolinhas que permitiram a observação da variabilidade espacial e que se mostraram de grande valia aos cafeicultores.

\section{AGRADECIMENTOS}

Ao Conselho Nacional de Desenvolvimento Científico e Tecnológico (CNPq), pelo aporte financeiro à realização deste trabalho.

\section{REFERÊNCIAS}

ALVES, M.C.; SILVA, F.M.; MORAES, J.C.; POZZA, E.A.; OLIVEIRA, M.S.; SOUZA, J.C.S.; ALVES, L.S. Geostatistical analysis of the spatial variation of the berry borer and leaf miner in a coffee agroecosystem. Precision Agriculture, Dordrecht, v.10, n.12, p.1-14, dez. 2009.

BLISKA, F.M.M.; VEGRO, C.L.R.; AFONSO JÚNIOR, P.C.; MOURÃO, E.A.B.; CARDOSO, C.H.S. Custos de produção de café nas principais regiões produtoras do Brasil. Informações Econômicas, São Paulo, v.29, n.8, p.5-20, ago. 2009.

BRASIL. Ministério da Agricultura, Pecuária e Abastecimento. Produção de grãos. Brasília, 2009. Disponível em: <http://www.agricultura.gov.br/>. Acesso em: 29 nov. 2009.

CARVALHO, G.R.; BOTELHO, C.E.; BARTHOLO, G.F.; PEREIRA, A.A.; NOGUEIRA, Â.M.; CARVALHO, A.M. de. Comportamento de progênies F4 obtidas por cruzamentos de 'Icatu' com 'Catimor'. Ciência e Agrotecnologia, Lavras, v.33, n.1, p.47-52, jan./fev. 2009.

FARACO, M.A.; URIBE-OPAZO, M.A.; SILVA, E.A.A.; JOHANN, J.A.; BORSSOI, J. Seleção de modelos de variabilidade espacial para elaboração de mapas temáticos de atributos físicos do solo e produtividade da soja. Revista Brasileira de Ciência do Solo, Viçosa, MG, v.32, n.2, p.463476, mar./abr. 2008.

FROGBROOK, Z.L.; OLIVER, M.A.; SALAHI, M.; ELLIS, R.H. Exploring the spatial relations between cereal yield and soil chemical properties and the implications for sampling. Soil Use and Management, Oxford, v.18, n.1, p.1-9, fev. 2002.

GOMES, F.P.; GARCIA, C.H. Estatística aplicada a experimentos agronômicos e florestais. Piracicaba: FEALQ, 2002. 305 p.

ISAAKS, E.H.; SRIVASTAVA, R.M. An introduction to applied geostatistics. New York: Oxford University, 1989. $561 \mathrm{p}$.

JOHANN, J.A.; SILVA, M.C.A.; URIBE-OPAZO, M.A.; DALPOSSO, G.H. Variabilidade espacial da rentabilidade, perdas na colheita e produtividade do feijoeiro. Engenharia Agrícola, Jaboticabal, v.30, n.4, p.700-714, jul/ago. 2010. 
KERRY, R.; OLIVER, M.A. Sampling requirements for variograms of soil properties computed by the method of moments and residual maximum likelihood. Geoderma, Amsterdam, v.140, p.383396, 2007.

MAPA, R.B.; KUMARAGAMAGE, D. Variability of soil properties in a tropical Alfisol used for shifting cultivation. Soil Technology, Amsterdam, v.9, p.187-197, 1996.

OLIVEIRA, E.; SILVA, F.M.; GUIMARÃES, R.J; SOUZA, Z.M. Eliminação de linhas em cafeeiros adensados por meio semimecanizado. Ciência e Agrotecnologia, Lavras, v.31, n.6, p.1.826-1.830, 2007a.

OLIVEIRA, E.; SILVA, F.M.; SALVADOR, N.; SOUZA, Z.M.; CHALFOUN, S.M.;

FIGUEIREDO, C.A.P. Custos operacionais da colheita mecanizada do cafeeiro. Pesquisa Agropecuária Brasileira, Brasília, v.42, n.6, p.827-831, 2007b.

PROCAFÉ. Fundação Procafé. Padrões referenciais médios para avaliação de resultados de análise de solos e análise foliar na cultura do café. Varginha, 2011. Disponível em:

<http://www.fundacaoprocafe.com.br/laboratorio/solos-e-folhas/padroes-referenciais >. Acesso em: 17 jun. 2011.

RIBEIRO JÚNIOR, P.J.; DIGGLE, P.J. GeoR: a package for geostatistical analysis. R-News, New York, v.1, n.2, p.14-18, 2001.

RIBEIRO, M.; LIMA, L. .; FARIA, F.H.S.; REZENDE, F.C.; FARIA, L.A. Efeitos de águas residuárias de café no crescimento vegetativo de cafeeiros em seu primeiro ano. Engenharia Agrícola, Jaboticabal, v.29, n.4, p.569-577, 2009.

SILVA, F.M.; ALVES, M.C.; SOUZA, J.C.S.; OLIVEIRA, M.S. Efeitos da colheita manual na bienalidade do cafeeiro em Ijaci, Minas Gerais. Ciência e Agrotecnologia, Lavras, v.34, n.3, p.625632, 2010.

SILVA, F.M.; SOUZA, Z.M.; FIGUEIREDO, C.A.P.; MARQUES, J.J.; MACHADO, R.V. Variabilidade espacial de atributos químicos e de produtividade na cultura do café. Ciência Rural, Santa Maria, v.37, n.2, p.401-407, 2007.

SILVA, F.M.; SOUZA, Z.M.; FIGUEIREDO, C.A.P.; VIEIRA, L.H.S.; OLIVEIRA, E. Variabilidade espacial de atributos químicos e produtividade da cultura do café em duas safras agrícolas. Ciência e Agrotecnologia, Lavras, v.32, n.1, p.231-241, 2008.

SILVA, V.R.; REICHERT, J.M.; STORCK, L.; FEIJO, S. Variabilidade espacial das características químicas do solo e produtividade de milho em um Argissolo Vermelho-Amarelo distrófico arênico. Revista Brasileira de Ciência do Solo, Viçosa-MG, v.27, n.6, p. 1.001-1.012, 2003.

TRABAQUINI, K.; MIGLIORANZA, É.; FRANÇA, V.; PEREIRA NETO, O. Uso da geotecnologia para caracterizar os cafezais no município de Londrina-PR, em relação à altimetria, declividade e tipo de solo. Revista Brasileira de Engenharia Agrícola, Jaboticabal, v.30, n.6, p.1.136-1.147, 2010 .

VIEIRA, S.R. Geoestatística em estudos de variabilidade espacial do solo. In: NOVAIS, R.F. de; ALVAREZ, V.H.; SCHAEFER, C.E.G.R. (Ed.). Tópicos em ciência do solo. Viçosa-MG:

Sociedade Brasileira de Ciência do Solo, 2000. v.1, p.1-54.

WEBSTER, R. Statistics to support soil research and their presentation. European Journal of Soil Science, Oxford, v.52, p.331- 340, 2001.

WEBSTER, R.; OLIVER, M. Geostatistics for environmental scientists. Chichester: John Wiley \& Sons, 2007. 315 p. 